\title{
Ubiquitous Healthcare Profile Management Applying Smart Card Technology
}

\author{
Maria-Anna Fengou, Georgios Mantas, Dimitrios Lymberopoulos and Nikos \\ Komninos \\ Wire Communications Laboratory, Electrical and Computer Engineering Department, \\ University of Patras \\ University Campus, 26504 Rio Patras, Greece \\ afengoum@upatras.gr, gman@upatras.gr, dlympero@upatras.gr, \\ nkome ieee.org
}

\begin{abstract}
Nowadays, the patient-centric healthcare approach is focused on ubiquitous healthcare services. Furthermore, the adoption of cloud computing technology leads to more efficient ubiquitous healthcare systems. Moreover, the personalization of the delivery of ubiquitous healthcare services is enabled with the introduction of user profiles. In this paper, we propose five generic healthcare profile structures corresponding to the main categories of the participating entities included in a typical ubiquitous healthcare system in a cloud computing environment. In addition, we propose a profile management system incorporating smart card technology to increase its efficiency and the quality of the provided services of the ubiquitous healthcare system.
\end{abstract}

Keywords: cloud computing, user profiles, profile management, smart card.

\section{Introduction}

Nowadays, the patient-centric healthcare approach is focused on healthcare services related to effective treatment, disease prevention, proactive actions and life quality improvement at the right time, right place and right manner without limitations on time and location. Essentially, the patient-centric approach can be materialized through ubiquitous healthcare. Furthermore, the current trend of outsourcing of IT infrastructure via adoption of cloud computing technology leads to more efficient ubiquitous healthcare systems in which end-user data are stored and processed at many different places distributed geographically [1]. However, many ubiquitous healthcare services built by third-party providers are usually tied to specific scopes and they are not able to be adapted to the unique preferences and interest of the endusers. Because of these limitations, personalized healthcare systems based on user profiles have been recently deployed [2]. Profile management materializes the main functionality of personalized healthcare systems [3]. In addition, profile management integrates the user profiling process consisting of three main steps. In the first step, an information collection process takes place in order raw information related to the user to be gathered. The second phase includes the user profile construction process based on the gathered user information. Finally, in the third phase, a service makes use of the data in the user profile in order to provide personalized services [4]. During the past few years, a lot of effort has been invested in user profile management systems for ubiquitous healthcare systems. However, these profile management systems incorporate user profiles including limited user information associated with user preferences and interests. Therefore, it is essential the creation of more enriched user profiles for efficient ubiquitous healthcare systems. Furthermore, taking into consideration the challenges that a ubiquitous healthcare 
system should face in order to provide high quality and reliable services, it is required the creation of profiles for all the participating entities of such a system.

Thus, in this paper, we propose five generic healthcare profile structures that correspond to the main categories of the participating entities of a typical ubiquitous healthcare system in a cloud computing environment. The main objective of these proposed structures is to contribute toward the creation of more enriched profiles for all participating entities of a ubiquitous healthcare system. Additionally, we propose a profile management system incorporating smart card technology in order to increase its efficiency and the quality of the provided services of the ubiquitous healthcare system. Especially, smart cards are proposed to include information referencing to large amount of stored profile data for the creation of enriched user profiles. Profile data should always be available, over all networks, from all supported services enabling service continuity and optimal user experience.

Following the introduction, this paper is organized as follows. In Section 2, the related work of ubiquitous healthcare sector in cloud computing environment and profile management is presented. Furthermore, a brief overview of smart card technology is given. In Section 3, the proposed five generic healthcare profile structures are described. In Section 4, the proposed profile management system is discussed. Finally, Section 5 concludes the paper.

\section{Related Work}

\subsection{Ubiquitous Healthcare in Cloud Computing}

Several platforms have been proposed that combine cloud computing and network security concepts implemented in the healthcare domain [1]. MoCAsH [5] is an infrastructure for assistive healthcare. It inherits the advantages of Cloud computing and embraces concepts of mobile sensing, active sensor records, and collaborative planning. The work [6] proposes a system designed and implemented using the cloud computing platform to provide a long term offsite medical image archive solution. Phynx [7] presents the development of an open source software solution that supports management and clinical review of patient data from electronic medical records databases or claims databases for pharmacoepidemiological drug safety studies. In [8], it is proposed a solution to automate the existing processes for patients' vital data collection that require a great deal of labor work to collect, input and analyze the information. This work is also based on the concepts of utility computing and wireless sensor networks. The information becomes available in the "cloud" from where it can be processed by expert systems and/or distributed to medical staff. Finally, [9] presents the implementation of a mobile system that enables electronic healthcare data storage, update and retrieval using cloud computing. The mobile application provides management of patient health records and medical images.

\subsection{Profile Management System}

Personalization and user profile management holds the promise of improving the uptake of new technologies and allowing greater access to their benefits $[10,11]$. In a typical profile management scenario, there are multiple profile storage locations. Many of these locations will not store the total profile but only components that apply to a device, an application, a network function or service. Different locations may have different persistence and priority levels. Although the user profile data is distributed amongst a range of devices, applications and services, ideally, all profile data should always be 
available, over all networks, from all supported devices and services, including fixed and mobile services allowing service continuity and optimal user experience [11].

The work [12] proposes a user profile management that allows the creation of an instance of the user profile for each application and for each instance of context. Major enhancement consists in the enrichment of the user profile with dynamic data permitting to characterize the user environment, the serving device and network. Sutterer et al. [13] introduce a user profile management approach for managing and delivering contextdependent user profiles for several applications and decouples the application development from context processing in ubiquitous computing environments.

\subsection{Smart Card Technology}

Smart cards are plastic pocket-sized cards embedded with either a microcontroller chip or only a memory chip with non - programmable logic. Nowadays, smart cards are used in a variety of applications ranging to identify individuals and get cash from the bank to mobile telecommunications (SIM-cards). Smart cards are favorable for applications that require data confidentiality and data integrity. Data confidentiality guarantees that the data/information contained on the card is accessible only by legitimate users. Data integrity ensures that the data/information stored on the card is always defined and is not changed even if the power to the card is removed during a computation involving data stored on the card.

The operating systems used in the majority of current smart cards are based on the provisions of the ISO/IEC 7816 family of standards. The smart card operating systems are stored in the ROM of the chip. Moreover, the available part of RAM and a small part of EEPROM are used by the operating systems. They implement a standard set of commands (e.g. 20-30) which the card can "understand" [14].

\section{Proposed Healthcare Profile Structures}

The electronic Medical Health Record (eMHR) is considered as the core component for ubiquitous healthcare systems. Treatments, demographic, examinations and other medical information of each citizen are stored in eMHRs. However, to deliver personalized ubiquitous healthcare services, it is also essential the incorporation of profiles of the participating entities in the ubiquitous healthcare systems.

In a ubiquitous healthcare system, there are the following categories of participating entities:

- Patient: around whom the healthcare services are built depending on his health condition and the services that he is interested on;

- Patient's social network: doctors, healthcare providers, family members, volunteers, etc;

- Hospital: in case the patient's health condition is critical, he will be transferred to a hospital;

- Smart Home: a home equipped with advanced technology (e.g. wireless sensor networks) adequate to provide safety and a quality of life to people suffering from chronic diseases (e.g. cardiac) or elders having dementia;

- Office: the area where the patient works;

- Vehicle: the personal vehicle that the patient uses to be transferred.

All these entities are either directly or indirectly related to the patient when his health condition is critical and a ubiquitous healthcare service should be delivered. Based on the features of these entities, we propose a profile structure for each entity. To generate the profile structure of each entity, we used [15] and the method described in [16]. Using this method, the basic steps for the generation of the profile structures are: sampling, 
analyzing and modeling. Thus, the proposed generic healthcare profile structures are the following:

- User Healthcare Profile: This profile corresponds to the Patient and Patient's Social Network categories;

- Hospital Profile: This profile corresponds to the Hospital category;

- Smart Home Profile: This profile corresponds to the Smart Home Profile category;

- Office Profile: This profile corresponds to the Office Profile; • Vehicle Profile: This profile corresponds to the Vehicle Profile.

The proposed five generic healthcare profile structures are depicted in Fig. 1:

\begin{tabular}{|c|}
\hline \multicolumn{1}{|c|}{ User Healthcare Profiles } \\
\hline Personal Information \\
\hline \\
\hline Preferences \\
\hline - $\quad$ Personal Preferences \\
- $\quad$ Provice Formaled Services Preferences \\
\hline Terminal Capabilities \\
\hline \\
\hline Required for Third Party \\
\hline \\
\hline Current Activity \\
\hline $\begin{array}{l}\text { This field allows the user to indicate what is } \\
\text { currently doing }\end{array}$ \\
\hline Current Context Info \\
\hline - $\quad$ Environmental Context \\
• Social Context \\
\hline User History \\
\hline $\begin{array}{l}\text { This field may contain a URI that indicates where } \\
\text { the user's history is stored }\end{array}$ \\
\hline Rules \\
\hline $\begin{array}{l}\text { This field contains statements that use as input } \\
\text { variable information stored in the profile }\end{array}$ \\
\hline Policies \\
\hline
\end{tabular}

\begin{tabular}{|l|}
\hline \multicolumn{1}{|c|}{ Office Profile } \\
\hline Identification Info \\
\hline \\
\hline Support Healthcare Services \\
\hline e.g. telemonitoring \\
\hline Available equipment facilities \\
\hline \\
\hline Available Office Members for support \\
\hline e.g. secretary \\
\hline Context Information \\
\hline Environmental \\
\hline
\end{tabular}

\begin{tabular}{|c|}
\hline Healthcare Center Profile \\
\hline Identification Info \\
\hline Supported Healthcare Service \\
\hline Available equipment and facilities \\
\hline $\begin{array}{l}\text { - Ambulance } \\
\text { - Beds }\end{array}$ \\
\hline Available professional healthcare staff \\
\hline $\begin{array}{l}\text { - Doctors } \\
\text { - Nurses } \\
\text { - Secretaries } \\
\text { - IT supported staff } \\
\text { - Administrator Support Staff }\end{array}$ \\
\hline Healthcare Center Availability \\
\hline Statistics (Volume metrics) \\
\hline Smart Home Profile \\
\hline Identification Info \\
\hline Support Healthcare Services \\
\hline e.g. telemonitoring \\
\hline Available equipment facilities \\
\hline Available Home Members/residents for support \\
\hline Context Information \\
\hline - Environmental \\
\hline
\end{tabular}

\begin{tabular}{|l|}
\hline \multicolumn{2}{|c|}{ Vehicle Profile } \\
\hline Vehicle Features \\
\hline \\
\hline Available equipment and facilities \\
\hline \\
\hline Support Services \\
\hline \\
\hline Context Information \\
\hline$\bullet \quad$ Environmental \\
\hline
\end{tabular}

Fig. 1. Proposed Healthcare Profile Structures 


\section{Proposed Profile Management System}

Our proposed profile management system is applied on an e-Health tele-monitoring system in a cloud computing environment for providing monitoring services to patients suffering from chronic diseases remotely. We consider that the e-Health telemonitoring system integrates a wide spectrum of participating entities including patients, doctors, nurses, family members, a hospital, a smart home, an office and a vehicle. The participating patients, doctors, nurses and family members are considered as the users of the e-Health tele-monitoring system.

In addition, each participating entity has its own profile that follows the structure corresponding to one of our proposed healthcare profile structures according to the main category to which the participating entity belongs. Thus, the proposed profile management system makes use of a number of User Healthcare Profiles, a Hospital Profile, a Smart Home Profile, an Office Profile and a Vehicle Profile. The incorporated data of each profile are stored in distributed databases in the cloud computing environment where the e-Health tele-monitoring system works.

The proposed profile management system of the e-Health tele-monitoring system is presented in Fig. 2:

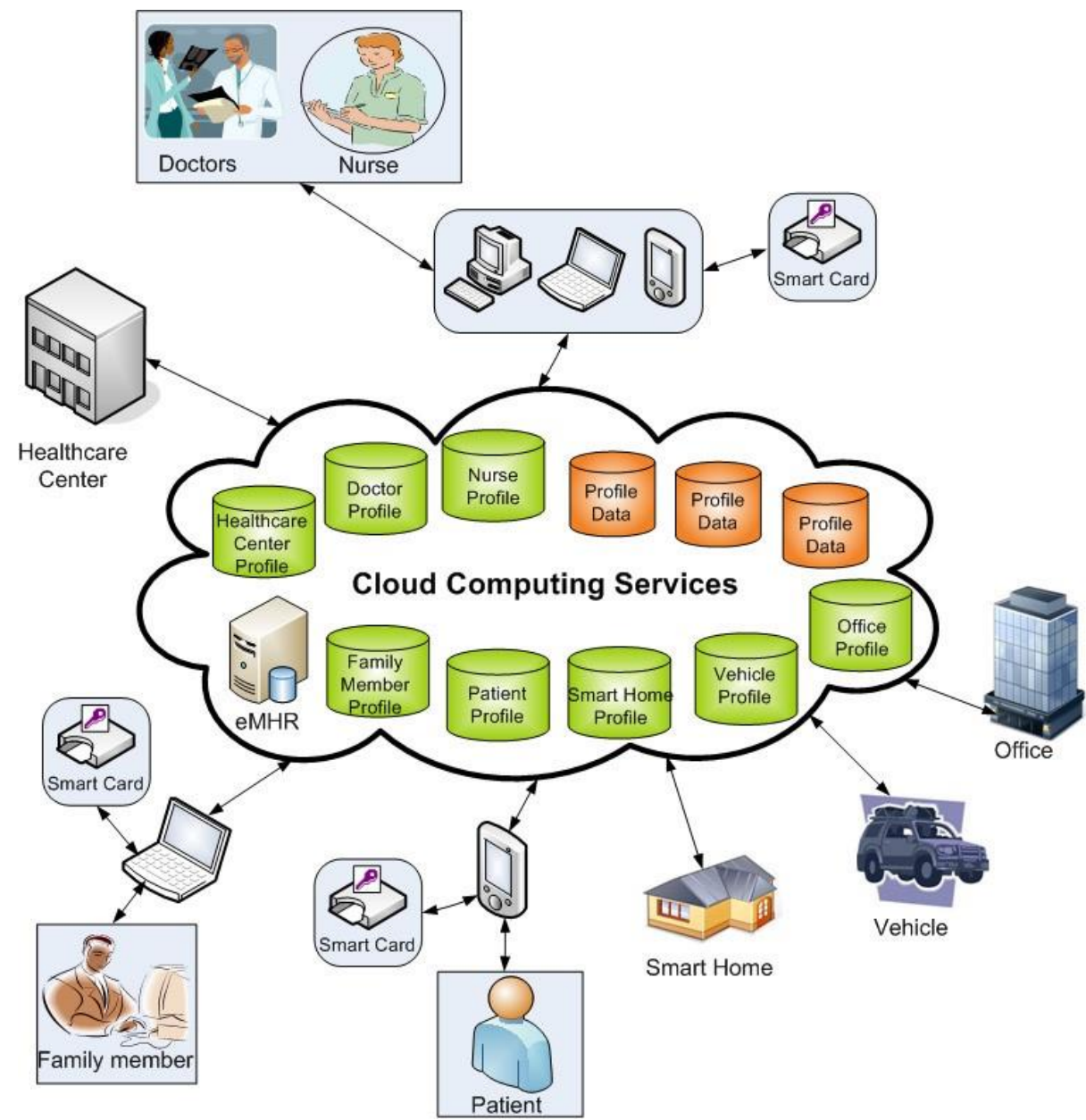

Fig. 2. Proposed Profile Management System

The patients are being monitored by the system in order any critical event to be detected timely. The main objective of the proposed profile management system is the creation of group profiles in order to provide high quality and reliable personalized healthcare services to the patients. 
The group profile is created based on the indexes stored in the User Healthcare Profiles. Each patient's profile contains an index to a group profile that is triggered whenever an event is detected. The events are related to the patient's health condition and they denote that the patient needs a medical advice or care due to aggravation of his/her health condition. The group is formed through certain management, scheduling and notification events.

Furthermore, in the context of the proposed profile management system, each one of the participating users poses a smart card storing information about the locations of databases where the corresponding profile data are stored. In other words, as it is depicted in Fig. 3, each user's smart card stores URL locations pointing to databases where large amount of profile data are found in order a User Healthcare Profiles to be created.

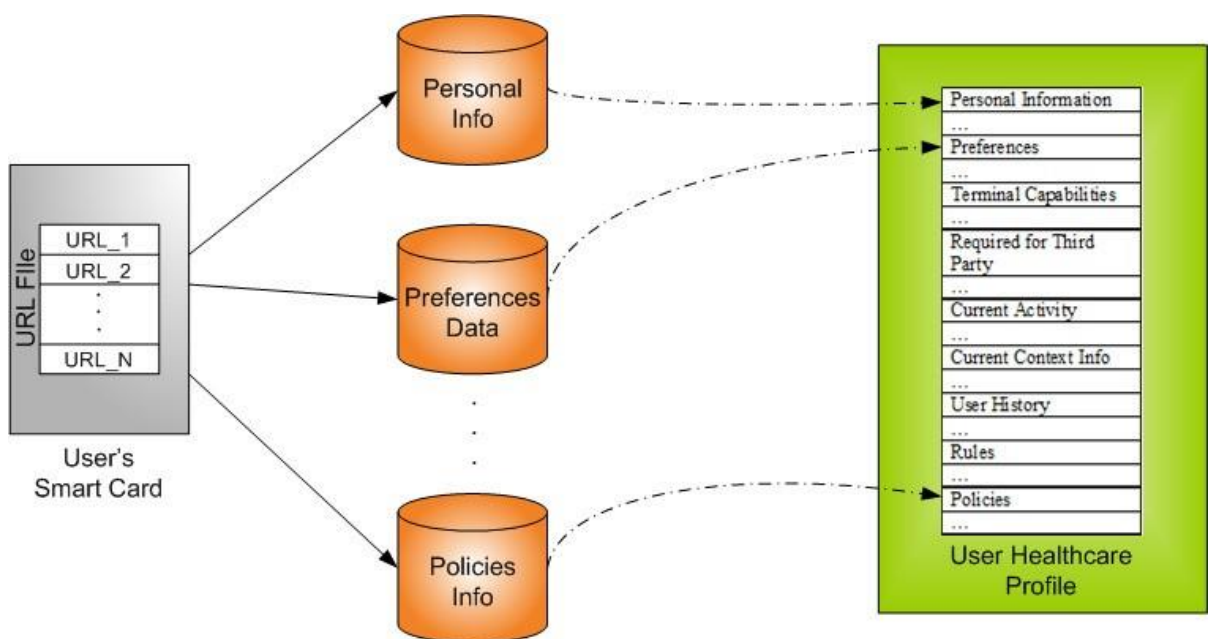

Fig. 3. User Healthcare Profiles in Smart Cards

\section{Conclusion and Future Work}

In this paper, we have proposed five generic healthcare profile structures for all the main categories of the participating entities of a typical ubiquitous healthcare system in a cloud computing environment. Our vision for these structures is to lead to more enriched profiles for all participating entities. Furthermore, we have proposed a profile management system integrating smart card technology to increase its efficiency and the quality of the provided services of the ubiquitous healthcare system. Finally, as future work, we intend to deploy the proposed profile management system for an e-Health tele-monitoring system in cloud computing environment.

\section{References}

1. Löhr, H., Sadeghi, A.R., Winandy, M.: Securing the E-Health Cloud. In: 1st ACM International Health Informatics Symposium, pp. 220--229, Virginia 2010

2. Guillén, S., Meneu, M.T., Serafin, R., Arredondo, M.T., Castellano, E., Valdivieso, B.: eDisease Management. A system for the management of the chronic conditions. In: 32nd Annual International Conference of the IEEE EMBS, pp. 1041--1044, Buenos Aires 2010

3. ETSI ES 202746 V0.0.16: Human Factors (HF); Personalization and User Profile Management; User Profile Preferences and Information, 2010 
4. Gauch, S., Speretta, M., Chandramouli, A., Micarelli, A.: User profiles for personalized information access. In: Brusilovsky, P., Kosba, A., Nejdl, W. (eds), pp.54--89, SpringerVerlag Berlin, Heidelberg (2007)

5. Hoang, D.B., Lingfeng, C.: Mobile Cloud for Assistive Healthcare (MoCAsH). In: Services Computing Conference (APSCC), pp. 325--332, Hangzhou 2010

6. Teng, C.C., Mitchell, J., Walker, C., Swan, A., Davila, C., Howard, D., Needham, T.: A medical Image Archive Solution in the Cloud. In: Software Engineering and Service Sciences (ICSESS), pp. 431--434, Beijing 2010

7. Egbring, M., Kullak-Ublick, G.A., Russmann, S.: Phynx: an open source software solution supporting data management and web-based patient-level data review for drug safety studies in the general practice research database and other health care database. In: PDS, Volume 19, Issue 1, pp. 38--44 (2010)

8. Rolim, C.O., Koch, F.L., Westphall, C.B., Werner, J., Fracalossi, A., Salvador, G.S.: A Cloud Computing Solution for Patient's Data Collection in Health Care Institutions. In: Second International Conference on eHealth, Telemedicine, and Social Medicine, pp. 95-99, 2010

9. Doukas, C., Pliakas, T., Maglogiannis, I.: Mobile Healthcare Information Management utilizing Cloud Computing and Android OS. In: EMBS, pp. 1037--1040, Buenos Aires 2010

10.ETSI TS 102747 V1.1.1, Human Factors (HF); Personalization and User Profile Management; Architectural Framework, 2009

11.Kovacikova, T., Petersen, F., Pluke, M., Bartolomeo, G.: User Profile Management Integration with the Universal Communications Identifier Concept, In: $13^{\text {th }}$ WSEAS International Conference on Communications (2009)

12.Chellouche, S.A., Arnaud, J., Négru, D.: Flexible User Profile Management for ContextAware Ubiquitous Environments, In: $7^{\text {th }}$ IEEE conference on Consumer communications and etworking conference (CCNC), pp. 98--984, Las Vegas 2010

13.Sutterer, M., Droegehorn, O., K., Kassel Univ: User Profile Management on Service Platforms for Ubiquitous Computing Environments, In: $65^{\text {th }}$ Vehicular Technology Conference (VTC), Dublin 2007

14.Rankl, W., Effing, W.: Smart Card Handbook, In: Wiley (2003)

15.Draft ETSI ES 202642 V0.0.28, "Human factors (HF), eHealth; personalization of eHealth systems," 2010

16.Casas, R., Marin, R.B., Robinet, A., Delgado, A.R., Yarza, A.R., McGinn, J., Picking, R., Grout, V..: User Modelling in Ambient Intelligence for Elderly and Disabled People. In:

Miesenberger, K., et al. (eds.) ICCHP 2008. LNCS, vol. 5105, pp. 1148--1158. Springer, Heidelberg (2008) 